\title{
Radiation characteristics and optical properties of filamentous cyanobacterium Anabaena cylindrica
}

\author{
Ri-Liang Heng, Euntaek Lee, and Laurent Pilon* \\ Mechanical and Aerospace Engineering Department, Henry Samueli School of Engineering and Applied Science, \\ University of California, Los Angeles, 420 Westwood Plaza, Eng. IV 37-132, Los Angeles, California 90095, USA \\ ${ }^{*}$ Corresponding author: pilon@seas.ucla.edu
}

Received December 17, 2013; revised February 13, 2014; accepted February 18, 2014; posted February 19, 2014 (Doc. ID 203148); published March 27, 2014

\begin{abstract}
This study presents experimental measurements of the absorption and scattering cross sections and the spectral complex index of refraction of filamentous cyanobacteria. Filamentous heterocystous cyanobacterium Anabaena cylindrica was chosen as a model organism. Its filaments consisted of long chains of polydisperse cells. Their average mass scattering and absorption cross sections were measured from 400 to $750 \mathrm{~nm}$ at four different times during their batch growth in medium BG-11(-N) under 3000 lux of white fluorescent light. The effective real (or refraction index) and imaginary (or absorption index) parts of the complex index of refraction were retrieved using an inverse method based on a genetic algorithm. The microorganisms were modeled as infinitely long and randomly oriented volume-equivalent cylinders. The absorption index featured peaks corresponding to chlorophyll $a$ $(\mathrm{Chl} a$ ) at 436 and $676 \mathrm{~nm}$ and phycocyanin (PCCN) at $630 \mathrm{~nm}$ and a shoulder around $480 \mathrm{~nm}$, corresponding to photoprotective carotenoids. The absorption peaks of Chl $a$ and PCCN concentrations increased and the shoulder due to carotenoids decreased in response to photolimitation caused by biomass growth. Subsequent nitrogen limitation caused the PCCN absorption peak to decrease significantly due to degradation of PCCN as an endogenous source of nitrogen for nitrogenase maintenance and synthesis, as confirmed by increasing heterocyst differentiation. The results can be used for predicting and optimizing light transfer in photobioreactors for wastewater treatment and ammonia or biofuel production. (c) 2014 Optical Society of America
\end{abstract}

OCIS codes: $\quad$ (010.4450) Oceanic optics; (010.5620) Radiative transfer; (120.5820) Scattering measurements;

(290.7050) Turbid media; (100.3200) Inverse scattering; (120.4530) Optical constants.

http://dx.doi.org/10.1364/JOSAA.31.000836

\section{INTRODUCTION}

The prolonged reliance on fossil fuels for many human activities has resulted in an accumulation of greenhouse gases in the atmosphere leading to global climate change and rising sea levels [1]. Petroleum has been used to produce (i) gasoline for transportation, (ii) fertilizer for agriculture, and (iii) plastic materials. While these sectors are expected to grow steadily over the next two decades, the supply of fossil fuels remains finite and is being depleted at an increasing rate. Also, geopolitical instability in oil-rich regions is a major concern for many nations seeking to ensure their energy security. Hence, finding sustainable solutions to meet global demands for petrochemical products and mitigating the resulting environmental damage are key issues of our times.

The production of biofuels from microorganisms has gained significant attention in recent years as a sustainable source of energy [2,3]. Photosynthetic microorganisms, such as green microalgae and cyanobacteria, can convert solar energy, water, and carbon dioxide $\left(\mathrm{CO}_{2}\right)$ into oxygen, carbohydrates, and lipids. Most of these microorganisms absorb solar energy in the photosynthetically active radiative (PAR) region defined by wavelengths ranging from 400 to $700 \mathrm{~nm}$. The lipids accumulated in their cells are extracted and converted into biodiesel [2]. What's more, the production of biodiesel is carbonneutral and compatible with existing infrastructure and vehicle technologies. Under specific growth conditions, certain species of green microalgae and cyanobacteria are also capable of producing hydrogen [4] and other added-value products, such as ammonium for fertilizers [5,6] and omega-3 fatty acids for nutritional supplements [5]. Finally, some microorganisms are able to remove phosphates and nitrates from effluent water by active or passive adsorption [5]. This represents a potentially cleaner and less energy-intensive wastewater treatment process than traditional methods [5].

Photosynthetic microorganisms are typically cultivated in either open pond systems or closed photobioreactors (PBRs) [2]. Poor light utilization and efficiency in these photobioreactors result in low production rate hampering the economic viability of the technology [3]. Indeed, photosynthetic microorganisms require an optimal level of irradiance to achieve their maximum photosynthetic rate. These versatile microorganisms are able to compensate for nonoptimal irradiance conditions by adjusting their different pigment concentrations via a process called photoacclimation [7]]. However, this natural adaptation strategy has its limits. When exposed to excessive irradiance, photosynthetic microorganisms suffer from photoinhibition due to photo-oxidative damage suffered by their light-harvesting antennae [8]. On the other hand, light penetration in the photobioreactor becomes severely limited at high biomass concentrations due to strong absorption and possibly self-shading [3]. Then, photosynthetic microorganisms are exposed to insufficient irradiance resulting in reduced photosynthetic activities, biomass growth, and added-value biomolecule production [3] .

Photobioreactors can be optimized to increase ammonia, hydrogen, and/or lipid production rates through light transfer 
modeling coupled with growth kinetics [3, $\underline{9}]$. To do so, spectral radiation characteristics are necessary parameters. However, measuring them can be costly and time consuming. Instead, they could be numerically predicted more easily based on the microorganism size distribution and complex index of refraction. This approach has been applied to spherical single cell microorganisms based on Lorenz-Mie theory [3] . However, some cyanobacteria are filamentous and cannot be treated as spherical [10]. The goal of this study is to develop an inverse method to retrieve the spectral real (refraction index) and imaginary (absorption index) parts of the complex index of refraction of filamentous cyanobacteria. A. cylindrica was chosen as a model organisms and its radiation characteristics were measured.

\section{BACKGROUND}

\section{A. Light Transfer}

In order to design efficient PBRs, it is necessary to predict the local radiation intensity therein [3]. Light transfer in a homogeneous absorbing, scattering, and nonemitting turbid medium is governed by the radiative transfer equation (RTE) written as [11]

$$
\begin{aligned}
\hat{\mathbf{s}} \cdot \nabla I_{\lambda}(\mathbf{r}, \hat{\mathbf{s}})= & -\kappa_{\lambda} I_{\lambda}(\mathbf{r}, \hat{\mathbf{s}})-\sigma_{s, \lambda} I_{\lambda}(\mathbf{r}, \hat{\mathbf{s}}) \\
& +\frac{\sigma_{s, \lambda}}{4 \pi} \int_{4 \pi} I_{\lambda}(\mathbf{r}, \hat{\mathbf{s}}) \Phi_{T, \lambda}\left(\hat{\mathbf{s}}_{i}, \hat{\mathbf{s}}\right) \mathrm{d} \Omega_{i},
\end{aligned}
$$

where $I_{\lambda}$ is the spectral radiation intensity about the direction $\hat{\mathbf{s}}$ at location $\mathbf{r}$ (in $\mathrm{W} / \mathrm{m}^{2} \cdot \mathrm{sr} \cdot \mathrm{nm}$ ), while $\kappa_{\lambda}$ and $\sigma_{s, \lambda}$ are the spectral absorption and scattering coefficients in $\left(\mathrm{m}^{-1}\right)$, respectively. The first and second terms on the right-hand side of Eq. (1) represent, respectively, the attenuation by absorption and outscattering, while the last term corresponds to the augmentation of radiation due to inscattering. The average absorption and scattering coefficients can be expressed in terms of the average mass absorption, $\bar{A}_{\text {abs }, \lambda}$, and scattering, $\bar{S}_{\text {sca }, \lambda}$, cross sections (in $\mathrm{m}^{2} / \mathrm{kg}$ ) as [12]

$$
\kappa_{\lambda}=\bar{A}_{\mathrm{abs}, \lambda} X \quad \text { and } \quad \sigma_{s, \lambda}=\bar{S}_{\mathrm{sca}, \lambda} X,
$$

where $X$ is the microorganism dry mass concentration expressed in $\mathrm{kg}$ of dry weight $/ \mathrm{m}^{3}$ of suspension $\left(1 \mathrm{~kg} / \mathrm{m}^{3}=1\right.$ in $\mathrm{g} / \mathrm{L})$. In addition, $\beta_{\lambda}$ is the so-called spectral extinction coefficient, defined as $\beta_{\lambda}=\kappa_{\lambda}+\sigma_{s, \lambda}$. The scattering phase function, $\Phi_{T, \lambda}\left(\hat{\mathbf{s}}_{i}, \hat{\mathbf{s}}\right)$, represents the probability that radiation propagating in the solid angle, $d \Omega_{i}$, around direction $\hat{\mathbf{s}}_{i}$ be scattered into the solid angle, $d \Omega$, along the direction $\hat{\mathbf{s}}$. It is normalized such that

$$
\frac{1}{4 \pi} \int_{4 \pi} \Phi_{T, \lambda}\left(\hat{\mathbf{s}}_{i}, \hat{\mathbf{s}}\right) \mathrm{d} \Omega_{i}=1 .
$$

Finally, the asymmetry factor for an axisymmetric phase function is defined as [3]

$$
g_{\lambda}=\frac{1}{2} \int_{0}^{\pi} \Phi_{T, \lambda}(\theta) \cos \theta \sin \theta \mathrm{d} \theta,
$$

where $\theta$ is the scattering angle between directions $\hat{\mathbf{s}}_{i}$ and $\hat{\mathbf{s}}$.

\section{B. Radiation Characteristics of Photosynthetic Microorganisms}

The radiation characteristics of photosynthetic microorganisms in suspension can be obtained either through experimental measurements [12-14] or numerical simulations [15-17]. Most efforts have focused on quasi-spherical cells such as the green microalgae $C$. reinhardtii $[\underline{13}, \underline{17}]$. Pilon and coworkers experimentally measured the radiation characteristics of several photosynthetic $H_{2}$ producing microorganisms $[\underline{12}, 13]$ and of lipid producing green microalgae $[\underline{14}, \underline{18}]$. The absorption, $\kappa_{\lambda}$, and scattering, $\sigma_{s, \lambda}$ coefficients of these microorganisms were normalized either by their cell number densities, $N_{T}$, in $\left(/ \mathrm{m}^{3}\right)$ [14] or by their dry mass concentrations, $X[\underline{12}, \underline{13}]$, according to Eq. (2). The cell number density, $N_{T}$, of single-cell microorganisms can be easily measured. However, filamentous cyanobacteria consist of long chains of cells. Defining their number density, $N_{T}$, becomes difficult as both the number of cells and filaments must be considered for an accurate characterization of their morphology. Therefore, the absorption, $\kappa_{\lambda}$, and scattering, $\sigma_{s, \lambda}$, coefficients of filamentous cyanobacteria should be normalized by their dry mass concentration, $X$, as performed for Anabaena variabilis [12].

Moreover, microorganisms are typically modeled as scattering particles with time-invariant radiation characteristics $[3, \underline{9}, \underline{19}]$. The latter are often measured only once during the entire growth, typically during the exponential phase [12-15,20]. However, microalgae and cyanobacteria undergo changes in pigment concentration as well as changes in their lipid, protein, and carbohydrate content in response to photoadaptation [7] or nutrient limitation [21]. These changes in the cell composition may strongly affect their radiation characteristics [22].

\section{Anabaena cylindrica}

The freshwater cyanobacterium Anabaena cylindrica belongs to a genus known for its ability to fix nitrogen and produce ammonia using the enzyme nitrogenase contained in differentiated cells called heterocysts [23-25]. The Anabaena genus has been used to remove nitrates and phosphates from contaminated waters as well as to produce fertilizers by photosynthesis and fixation of atmospheric nitrogen [6]. In addition, despite its low lipid content of about 5 dry wt. \% [26], A. cylindrica has also been considered for biodiesel production due to its large cell size and its filamentous morphology requiring less energy intensive harvesting methods, such as filtration, to separate the biomass from the growth medium.

Figure 1 shows a micrograph of the heterocystous filamentous A. cylindrica consisting of connected and nearly spherical vegetative cells and fewer and larger heterocysts $2-4 \mu \mathrm{m}$ in diameter. Filaments' length varies widely but typically exceeds $100 \mu \mathrm{m}$. In response to nitrogen limitation, $A$. cylindrica increases its heterocyst differentiation rate and nitrogenase activity [27]. It also adapts its pigment concentrations. A. cylindrica possesses the pigments chlorophyll $a$ (Chl a) and accessory pigments phycocyanin (PCCN) and carotenoids [28]. They enable it to absorb over different spectral bands of the PAR region for a more efficient utilization of solar energy. In fact, the in vivo absorption peaks of these pigments are distinct with Chl $a$ absorbing most strongly around 436 and $676 \mathrm{~nm}$ [29], PCCN around $630 \mathrm{~nm}$ [30], 


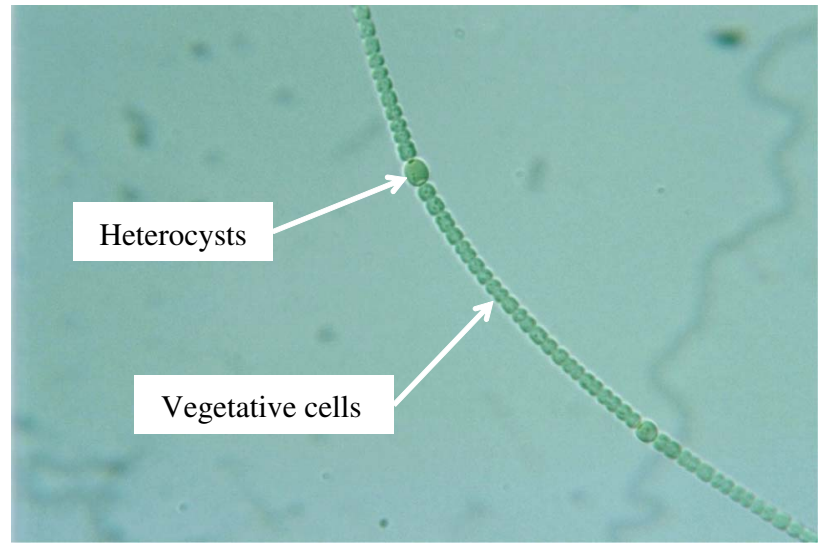

Fig. 1. Micrograph image of A. cylindrica showing vegetative cells and heterocysts $2.5-3 \mu \mathrm{m}$ in diameter. Reproduced with permission from Prof. Yuuji Tsukii, Hosei University, Protist Information Server http://protist.i.hosei.ac.jp.

and photosynthetic (PSC) and photoprotective (PPC) carotenoids between 400 and $550 \mathrm{~nm}$ [29].

\section{Optical Properties of Photosynthetic Microorganisms} Quirantes and Bernard [16] modeled algal cells as two-layer spherical particles with an inner core and an external coating representing the enveloped organelles and the cell membrane, respectively. The external layer was nonabsorbing and had a refraction index of 1.36. The inner core was absorbing and had a complex index of refraction of $1.40+i 0.005$ [16]. The authors found that the difference between the absorption and scattering efficiency factors of the heterogeneous coated sphere and of a homogeneous volume-equivalent sphere with a volume-averaged complex index of refraction was negligible. These results suggest that heterogeneous microorganism cells can be treated as homogeneous with some effective complex index of refraction. This approach has been widely used in the ocean optics community $[15,22]$.

Lee et al. [10] developed and validated an inverse method combining the Lorenz-Mie theory for spherical particles with a genetic algorithm [31] to retrieve the effective complex index of refraction of various microalgae by treating them as homogeneous polydisperse spheres. The authors also compared the absorption and scattering cross sections of spheroidal cells predicted by the T-matrix method based on the major and minor diameter distributions measured experimentally and those predicted by Lorenz-Mie theory for spherical particles with identical surface area and complex index of refraction. Their results demonstrated that spheroidal cells with aspect ratio less than 1.33 could be treated as spherical cells.

Moreover, Lee and Pilon [32] reported that the values of absorption and scattering cross sections per unit length of a randomly oriented linear chain of optically soft and monodisperse spheres of diameter $d_{s}$ converge asymptotically toward that of a randomly oriented and infinitely long cylinder with volume-equivalent diameter $d_{c, e q}=\sqrt{(2 / 3)} d_{s}$ and the same complex index of refraction, provided that the chain was sufficiently long. The minimum length required to approximate a randomly oriented linear chain of spheres as a randomly oriented and infinitely long cylinder decreased as the size parameter, $\chi_{s}=\pi d_{s} / \lambda$ increased. In fact, for size parameter and complex index of refraction representative of photosynthetic microorganisms, 15 spheres were sufficient.
In addition, randomly oriented linear chains of polydisperse spheres, with size distribution representative of $A$. cylindrica, were shown to have similar absorption and scattering cross sections per unit length as their volume-equivalent cylinders.

The present study aims to retrieve the spectral effective complex index of refraction of cyanobacterium A. cylindrica by treating its filaments of vegetative cells and heterocysts as infinitely long and randomly oriented cylinders. To do so, the scattering phase function $\Phi_{T, \lambda}\left(\hat{\mathbf{s}}_{i}, \hat{\mathbf{s}}\right)$, as well as the average mass absorption $\bar{A}_{\text {abs }, \lambda}$ and scattering $\bar{S}_{\text {sca }, \lambda}$ cross sections, were measured from 400 to $750 \mathrm{~nm}$ at different times during batch growth

\section{MATERIALS AND METHODS}

\section{A. Cultivation and Sample Preparation}

Heterocystous filamentous cyanobacterium A. cylindrica Lemmermann UTEX B1611 was purchased from UTEX Austin, Texas. It was cultivated in a BG-11(-N) medium in $250 \mathrm{ml}$ culture bottles fitted with vented caps and exposed to a continuous luminous flux of 2800-3000 lux (85-91 $\mu$ mol photon $/ \mathrm{m}^{2} \cdot \mathrm{s}$ ) provided by fluorescent light bulbs (GroLux by Sylvania, USA). The BG-11(-N) medium had the following composition (per liter of distilled water): $\mathrm{K}_{2} \mathrm{HPO}_{4} 40 \mathrm{mg}, \mathrm{MgSO}_{4} \cdot 7 \mathrm{H}_{2} \mathrm{O} 7.5 \mathrm{mg}, \mathrm{CaCl}_{2} \cdot 2 \mathrm{H}_{2} \mathrm{O} 36 \mathrm{mg}$, citric acid $\cdot \mathrm{H}_{2} \mathrm{O} 6 \mathrm{mg}$, ferric ammonium citrate $6 \mathrm{mg}$, $\mathrm{Na}{ }_{2}$ EDTA $\cdot 2 \mathrm{H}_{2} \mathrm{O} \quad 6 \mathrm{mg}, \mathrm{H}_{3} \mathrm{BO}_{3} 2.86 \mathrm{mg}, \quad \mathrm{MnCl}_{2} \cdot 4 \mathrm{H}_{2} \mathrm{O}$ $1.81 \mathrm{mg}, \mathrm{ZnSO}_{4} \cdot 7 \mathrm{H}_{2} \mathrm{O} 0.22 \mathrm{mg}, \mathrm{Na}_{2} \mathrm{MoO}_{4} \cdot 2 \mathrm{H}_{2} \mathrm{O} 0.39 \mathrm{mg}$, $\mathrm{CuSO}_{4} \cdot 5 \mathrm{H}_{2} \mathrm{O} 0.079 \mathrm{mg}$, and $\mathrm{Co}\left(\mathrm{NO}_{3}\right)_{2} \cdot 6 \mathrm{H}_{2} \mathrm{O} 0.0049 \mathrm{mg}$. This medium was recommended by UTEX and similar irradiance has been used in Ref. [25].

Nutrient availability in the medium can be estimated by elemental analysis [18]. The complete elemental composition of $A$. cylindrica was not available in the literature. However, the elemental composition does not vary significantly between species of the same genus [33,34]. The nitrogen content of $A$. cylindrica was reported to be $5 \%-10.8 \%$ by percentage of dry weight [35] while the cyanobacterium Anabaena flosaquae was reported to have 1 dry wt. \% of $K$ and 0.8 dry wt. \% of $P$ [36]. These values suggest that the culture of $A$. cylindrica in the BG-11(-N) medium would be limited in $P$ and $K$ at mass concentrations of 0.825 and $1.583 \mathrm{~kg} / \mathrm{m}^{3}$, respectively. On the other hand, the medium contained only traces of nitrates. It remains unclear whether the culture was nitrogen deficient, i.e., whether the rate of nitrogen uptake exceeded the rate of nitrogen-fixation.

The culture bottles were placed on an orbital shaker operated at $70 \mathrm{rpm}$ to ensure sufficient mixing to keep the microorganisms in suspension. Samples of $3 \mathrm{ml}$ were collected from the culture after $6,9,12$, and 14 days of growth to measure their radiation characteristics. These time intervals were selected to allow for sufficient cyanobacteria growth between measurements. Note that the culture started forming colonies after 15 days of batch growth after which experiments ended. To eliminate possible absorption and scattering by the growth medium, the suspension samples were centrifuged at $2000 \mathrm{rpm}(1300 \mathrm{~g})$ for $10 \mathrm{~min}$, washed twice with phosphate buffer saline (PBS) solution, and suspended in PBS [12,13, 18]. Smaller volumes of PBS were used to replace the growth medium in order to obtain larger biomass concentrations in the samples than in the culture. This was performed to obtain a higher signal-to-noise ratio in the transmission measurements. 
A calibration curve relating the optical density (OD) of the suspension at $750 \mathrm{~nm}$ to the dry mass concentration, $X$, was obtained prior to performing the radiation characteristics measurements. The OD was measured for five different mass concentrations of A. cylindrica in disposable polystyrene cuvettes with $1 \mathrm{~cm}$ pathlength using a UV-VIS spectrophotometer (Shimadzu, USA, Model UV-3103PC) [12,13,18]. The dry mass concentrations were obtained by filtering the cells with $0.45 \mathrm{~nm}$ pore size cellulose membrane filters (HAWP- 04700 by Millipore, USA) followed by overnight drying at $60^{\circ} \mathrm{C}$ in a vacuum oven [18]. The dried filters with the dry cells were weighed immediately after being removed from the oven using a precision balance (model AT261 by Delta Range Factory, USA) with a $0.01 \mathrm{mg}$ precision [18]. The mass concentration was related to the $\mathrm{OD}$ according to

$$
X=0.816 \mathrm{OD}_{750}
$$

with a coefficient of determination $R^{2}=0.96$.

\section{B. Cell Morphology}

Micrographs of A. cylindrica filaments were captured with a Leica LMIL microscope (Leica Microsystems, Illinois, USA) connected to a CCD camera (Spot Insight Model 4.2, MI, USA). The cells constituting the filamentous cyanobacterium were assumed to be spherical. We considered $N_{f}$ filaments and measured the diameter $\left(d_{s, i, j}\right)_{1 \leq j \leq N_{c}}$ of $N_{c}$ cells for each filament " $i$ ". The cell diameter was manually measured using the image analysis software ImageJ and taken as the maximum width across the cell in the direction perpendicular to the filament axis. For each of the four samples, at least 80 different $A$. cylindrica filaments were selected at random and the diameters of more than 14 cells per filament were measured, i.e., $N_{f}=80$ and $N_{c}=14$. Approximately 13 vegetative cells and 1 heterocyst were chosen randomly from each filament. This heterocyst frequency, defined as the ratio of the number of heterocysts to the total number of cells, was representative of the population of $A$. cylindrica in the samples.

As previously discussed, $A$. cylindrica filaments can be treated, as a first order approximation, as infinitely long cylinders given (i) their large size parameter, $\chi_{s} \geq 10$, (ii) their filamentous morphology, (iii) the quasi-sphericity of their heterocysts and vegetative cells, and (iv) their relatively narrow size distribution. The diameter, $d_{c, e q, i}$, of the volume-equivalent cylinder of filament " $i$ " can be expressed in terms of individual cell diameters as

$$
d_{c, e q, i}^{2}=\frac{\sum_{j=1}^{N_{c}} \frac{2}{3} d_{s, i, j}^{3}}{\sum_{j=1}^{N_{c}} d_{s, i, j}} \quad \text { with } i=1,2, \ldots, N_{f}
$$

These measurements led to the cylinder size distribution, $f\left(d_{c, e q}\right)$, defined as the number of equivalent cylinders having diameters between $d_{c, e q}$ and $d_{c, e q}+\mathrm{d} d_{c, e q}$.

\section{Radiation Characteristics Measurements}

\section{Assumptions}

The following assumptions were made in measuring the average mass absorption and scattering cross sections and the scattering phase function of $A$. cylindrica: (1) the microorganisms were well mixed and randomly oriented, (2) single scattering prevailed due to the low biomass concentrations considered, (3) the scattering phase function had azimuthal symmetry and was only a function of polar angle, $\theta$, (4) the scattering phase function was assumed to be constant over the PAR region [13], and (5) the scattering cross section of the cyanobacteria at $750 \mathrm{~nm}$ remains constant throughout the growth phase. The latter assumption constitutes the basis for measuring the temporal evolution of mass concentration $X$ from OD measurements and Eq. (5).

\section{Scattering Phase Function}

The scattering phase function was measured with a polar nephelometer at $633 \mathrm{~nm}$. The experimental setup, data analysis, and validation with latex microspheres and glass fibers were reported in detail by Berberoğlu and Pilon [12,13] and need not be repeated. Due to obstruction from the probe, scattering angles, $\theta$, beyond $170^{\circ}$ in the backward direction were not reported.

\section{Absorption and Scattering Coefficients}

Normal-normal transmittance measurements were performed in $1 \mathrm{~cm}$ pathlength cuvettes using a UV-VIS spectrophotometer (Shimadzu, USA, Model UV-3103PC) from 400 to $750 \mathrm{~nm}$ with $1 \mathrm{~nm}$ spectral resolution. The extinction coefficient of the suspension, $\beta_{\lambda}$, was obtained from these measurements after correcting for forward scattering by the suspension [13]. Indeed, A. cylindrica scatters radiation mainly in the forward direction, due to its large size parameter. A fraction of this forwardly scattered radiation was collected by the detector through its finite acceptance angle. It can be estimated from the previously measured scattering phase function and subtracted from the measured transmitted signal [13,37].

The absorption coefficient, $\kappa_{\lambda}$, was determined from normal-hemispherical transmittance measurements performed with an integrating sphere (ISR-3100 by Shimadzu, USA) and the above mentioned spectrophotometer at wavelengths from 400 to $750 \mathrm{~nm}$ [12]. However, imperfect reflections in the integrating sphere and the geometry of the setup prevented a portion of the scattered radiation from being collected by the detector. Thus, the measurements were corrected based on the fact that $A$. cylindrica does not absorb at $750 \mathrm{~nm} \mathrm{[38]}$ to obtain the absorption coefficient of the suspension, $\kappa_{\lambda}$, according to the procedure described in Refs. [13,37]. Finally, the scattering coefficient was obtained from $\sigma_{s, \lambda}=\beta_{\lambda}-\kappa_{\lambda}$.

Absorption and scattering coefficients were normalized by the mass concentration, $X$, estimated from OD measurements at $750 \mathrm{~nm}$ to obtain the average mass absorption and scattering cross sections $\bar{A}_{\mathrm{abs}, \lambda}$ and $\bar{S}_{\mathrm{sca}, \lambda}$ according to Eq. (2). Measurements were performed for three different mass concentrations on day $6,9,12$, and 14 . This was meant to assess experimental repeatability as well as to estimate the experimental uncertainties associated with $\bar{A}_{\mathrm{abs}, \lambda}$ and $\bar{S}_{\text {sca }, \lambda}$.

\section{Effective Optical Properties of Filamentous Cyanobacteria}

The average absorption and scattering cross sections, $\bar{C}_{\mathrm{abs}, \lambda}^{\prime}$ and $\bar{C}_{\text {sca }, \lambda}^{\prime}$, per unit length of filament (in $\mathrm{m}^{2} / \mathrm{m}$ ) can be determined from the measured average mass absorption and scattering cross sections, $\bar{A}_{\text {abs }, \lambda}$ and $\bar{S}_{\text {sca }, \lambda}$, using the following relationships adapted to filamentous cyanobacteria from Ref. [17], 


$$
\begin{aligned}
\bar{C}_{\mathrm{abs}, \lambda}^{\prime} & =\bar{A}_{\mathrm{abs}, \lambda} \rho_{d m}\left(1-x_{w}\right)\left(\frac{\pi}{6 N_{f}} \sum_{i=1}^{N_{f}} \sum_{j=1}^{N_{c}} d_{s, i, j}^{2}\right), \\
\text { and } \quad \bar{C}_{\mathrm{sca}, \lambda}^{\prime} & =\bar{S}_{\mathrm{sca}, \lambda} \rho_{d m}\left(1-x_{w}\right)\left(\frac{\pi}{6 N_{f}} \sum_{i=1}^{N_{f}} \sum_{j=1}^{N_{c}} d_{s, i, j}^{2}\right) .
\end{aligned}
$$

Here, $\rho_{d m}$ is the dry mass density of the microorganism taken as $1350 \mathrm{~kg} / \mathrm{m}^{3}$ [39]. The average volume fraction of water in the cells, denoted by $x_{w}$, was taken as either 0.75 or 0.85 which are considered to be the limits of its observed range [39]. The last term in parenthesis represents the average volume per unit length of the filaments of A. cylindrica.

The approach developed by Lee et al. [10] for spherical polydisperse scatterers was extended to retrieve the spectral refraction index, $n_{\lambda}$, and absorption index, $k_{\lambda}$, of filamentous cyanobacteria. Filaments were assumed to be randomly oriented, homogeneous, and infinitely long cylinders with volume-equivalent diameter distribution $f\left(d_{c, e q}\right)$. Figure $\underline{2}$ presents the schematic diagram used to retrieve $n_{\lambda}$ and $\overline{k_{\lambda}}$ of A. cylindrica from (i) the wavelength, (ii) the spectral refraction index $n_{\lambda, P B S}$ of the surrounding PBS medium reported in [40], (iii) the volume-equivalent cylinder diameter distribution $f\left(d_{c, e q}\right)$ of $A$. cylindrica, and (iv) the average absorption and scattering cross sections per unit length, $\bar{C}_{\text {abs }, \lambda}^{\prime}$ and $\bar{C}_{\text {sca }, \lambda}^{\prime}$, determined from experimental measurements of $\bar{A}_{\mathrm{abs}, \lambda}$ and $\bar{S}_{\text {sca, } \lambda}$ using Eq. (7). The electromagnetic wave theory predicting the absorption and scattering cross sections of infinitely long cylinders $[\underline{41}, \underline{42}]$ was used as the forward method. The objective function, $\delta_{\lambda}$, for each wavelength was defined as the sum of squared relative errors between the predicted average cross sections denoted by $\bar{C}_{\mathrm{abs}, \lambda, \mathrm{pred}}^{\prime}$ and $\bar{C}_{\mathrm{sca}, \lambda \text {,pred }}^{\prime}$ and those measured experimentally, $\bar{C}_{\mathrm{abs}, \lambda}^{\prime}$ and $\bar{C}_{\mathrm{sca}, \lambda}^{\prime}$, [Eq. ( $\left.(\underline{7})\right]$, i.e.,

$$
\delta_{\lambda}=\left(\frac{\bar{C}_{\mathrm{abs}, \lambda, \mathrm{ppred}}^{\prime}-\bar{C}_{\mathrm{abs}, \lambda}^{\prime}}{\bar{C}_{\mathrm{abs}, \lambda}^{\prime}}\right)^{2}+\left(\frac{\bar{C}_{\mathrm{sca}, \lambda, \mathrm{pred}}^{\prime}-\bar{C}_{\mathrm{sca}, \lambda}^{\prime}}{\bar{C}_{\mathrm{sca}, \lambda}^{\prime}}\right)^{2} .
$$

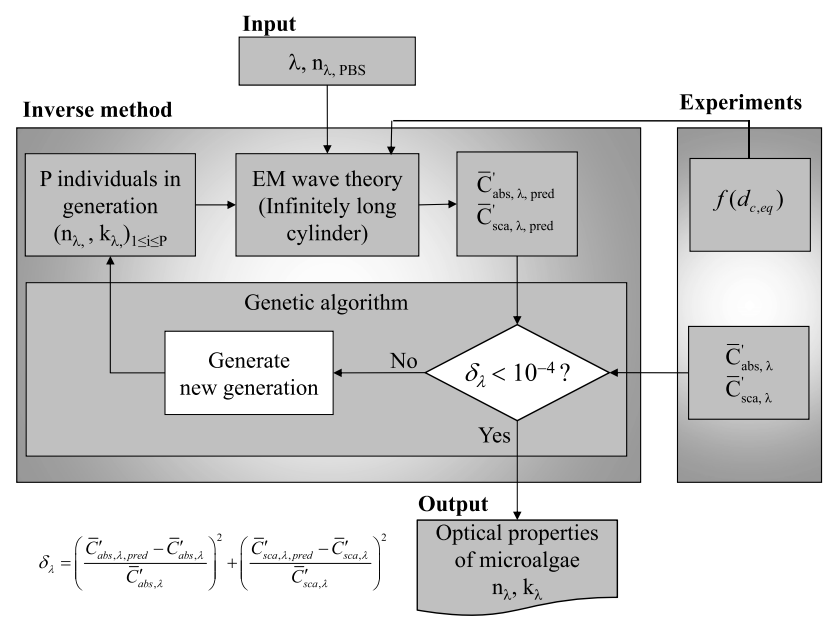

Fig. 2. Block diagram of the procedure used to retrieve the refraction index, $n_{\lambda}$, and absorption index, $k_{\lambda}$, from the measured absorption and scattering cross sections per unit length $\bar{C}_{\mathrm{abs}, \lambda}^{\prime}$ and $\bar{C}_{\mathrm{sca}, \lambda}^{\prime}$ at a given wavelength, $\lambda$, for volume-equivalent diameter distribution $f\left(d_{c, e q}\right)$. Here, $P=100$ individuals per generation for a maximum of 500 generations.
The genetic algorithm PIKAIA [31] was employed to find the pair $\left(n_{\lambda}, k_{\lambda}\right)$ that minimizes $\delta_{\lambda}$. PIKAIA was used with 500 generations and a population of 100 individuals per generation. The refraction and absorption indices, $n_{\lambda}$ and $k_{\lambda}$, were retrieved at 71 individual wavelengths between 400 and $750 \mathrm{~nm}$.

\section{RESULTS AND DISCUSSION}

Four samplings were performed on the 6th, 9th, 12th, and 14th day of A. cylindrica grown in batch mode. The corresponding mass concentrations were $X_{1}=0.086 \mathrm{~kg} / \mathrm{m}^{3}$, $X_{2}=0.108 \mathrm{~kg} / \mathrm{m}^{3}, X_{3}=0.151 \mathrm{~kg} / \mathrm{m}^{3}$, and $X_{4}=0.171 \mathrm{~kg} / \mathrm{m}^{3}$, respectively. Results from duplicate experiments fell within one another's experimental uncertainty. After 15 days of growth, the cyanobacterium culture started forming colonies and individual filaments could not be suspended as independent and randomly oriented scatterers.

Moreover, based on the previous elemental analysis, the biomass concentrations leading to $P$ and $K$ limitation were never reached, as the mass concentration did not exceed $X_{4}=0.171 \mathrm{~kg} / \mathrm{m}^{3}$. Thus, the cultures did not suffer from limitations in $P$ and $K$. Note that Brownell and Nicholas [43] achieved A. cylindrica mass concentrations ranging from 0.4 to $1.1 \mathrm{~kg} / \mathrm{m}^{3}$ after 13 days of growth in a nitrate deficient medium. These mass concentrations were significantly larger than the concentration reached in the present study. The difference can be attributed to the fact that the cyanobacteria in [43] were grown under an irradiance of 7000 lux instead of 3000 lux in the present study.

\section{A. Size Distribution and Heterocyst Frequency}

The volume-equivalent diameter distribution $f\left(d_{c, e q}\right)$ of $A$. $c y$ lindrica was estimated, based on Eq. (6), from more than $N_{f} \times$ $N_{c}=1120$ cells for each sample. Figures $3(\mathrm{a})-3(\mathrm{~d})$ show histograms of the volume-equivalent diameter distributions $f\left(d_{c, e q}\right)$ with bin size of $0.8 \mu \mathrm{m}$ for Samples $1,2,3$, and 4 and the mean diameter and standard deviation computed from diameter measurements of both vegetative cells and heterocysts. It is evident that the diameter distribution did not vary significantly from one sample to another. The mean diameter of the four samples fell within one standard deviation of one another and was equal to $2.7 \mu \mathrm{m}$.

Nitrogen limitation can affect the heterocyst frequency of A. cylindrica and therefore the size distribution since heterocysts tend to be larger than vegetative cells. Here, the heterocyst frequency was measured as $4.0 \%, 4.4 \%, 4.9 \%$, and $5.1 \%$ for Sample 1, 2, 3, and 4, respectively. This increase in heterocyst frequency and the composition of the BG-11(N) medium suggests that the culture suffered from nitrogen limitation from the beginning of the growth. However, this was not sufficient to affect the size distribution, $f\left(d_{c, e q}\right)$.

\section{B. Scattering Phase Function}

Figure $\underline{4}$ shows the experimentally measured scattering phase function of A. cylindrica at $633 \mathrm{~nm}$. Scattering by the microorganisms was strongly in the forward direction. For the sake of completeness, and for comparison with the literature, the asymmetry factor was estimated to be $g_{633}=0.985$ [Eq. (4)]. This large value was expected due to the large diameter and length of $A$. cylindrica filaments compared with the wavelength of light in the PAR region resulting in large size 

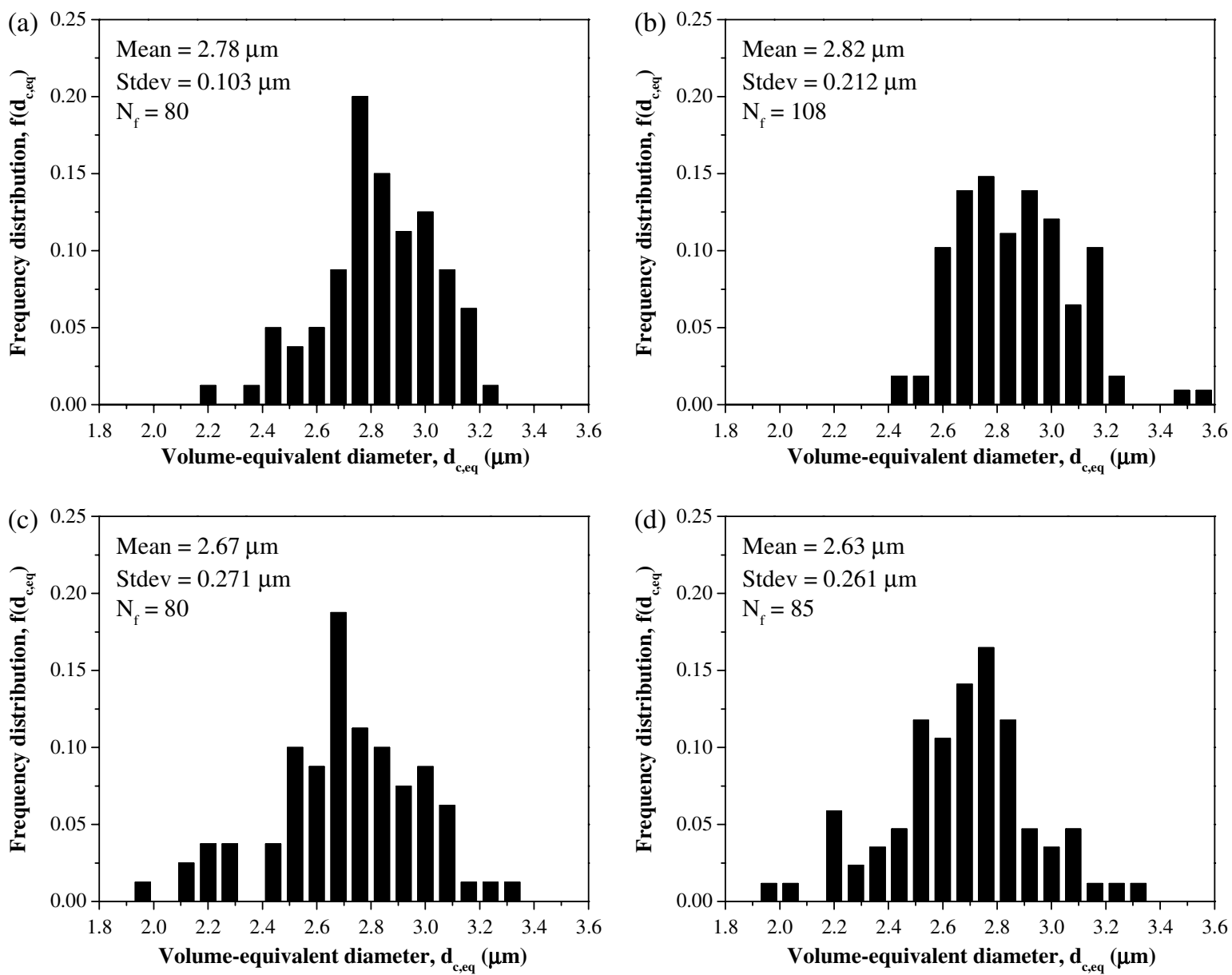

Fig. 3. Histograms of volume-equivalent diameter distribution $f\left(d_{\text {c.eq }}\right)$ of $A$. cylindrica filaments for Samples $1,2,3$, and 4 at concentrations (a) $X_{1}=0.086 \mathrm{~kg} / \mathrm{m}^{3}$, (b) $X_{2}=0.108 \mathrm{~kg} / \mathrm{m}^{3}$, (c) $X_{3}=0.151 \mathrm{~kg} / \mathrm{m}^{3}$, and (d) $X_{4}=0.171 \mathrm{~kg} / \mathrm{m}^{3}$ obtained from more than 1120 cells for each sample.

parameter $\chi_{s}$ in excess of 10 . Note that the asymmetry factor for $A$. variabilis was reported as $g_{633}=0.975$ [12].

\section{Mass Absorption and Scattering Cross Sections}

Figures 5(a) and 5(b), respectively, show the spectral absorption, $\kappa_{\lambda}$, and scattering, $\sigma_{s, \lambda}$, coefficients measured in the spectral range from 400 to $750 \mathrm{~nm}$ for Sample 3, collected after 12 days of growth for mass concentrations $X_{3,1}=0.431 \mathrm{~kg} / \mathrm{m}^{3}$, $X_{3,2}=0.296 \mathrm{~kg} / \mathrm{m}^{3}$, and $X_{3,3}=0.202 \mathrm{~kg} / \mathrm{m}^{3}$. These concentrations were achieved by suspending the cells in PBS from sample concentration $X_{3}=0.151 \mathrm{~kg} / \mathrm{m}^{3}$. Each data point represents the arithmetic mean of $\kappa_{\lambda}$ and $\sigma_{s, \lambda}$ measured three times for each concentration $\left(X_{3, i}\right)_{1 \leq i \leq 3}$, and the error bars correspond to $95 \%$ confidence interval. It is evident that the scattering and absorption coefficients increased with increasing mass concentrations. In addition, A. cylindrica absorbed mainly in the spectral region from 400 to $700 \mathrm{~nm}$ with peaks (i) at 436 and $676 \mathrm{~nm}$, corresponding to absorption by Chl $a$ [29]; (ii) at $630 \mathrm{~nm}$, corresponding to PCCN [30]; and (iii) a shoulder around $480 \mathrm{~nm}$, corresponding to absorption by PSC and PPC [29]. Small dips were observed in the scattering coefficient, $\sigma_{s, \lambda}$, around wavelengths corresponding to the absorption peaks.

Figures $\underline{5(\mathrm{c})}$ and $\underline{5(\mathrm{~d})}$ show the average mass absorption, $\bar{A}_{\text {abs }, \lambda}$, and scattering, $\bar{S}_{\text {sca }, \lambda}$, cross sections in the spectral range from 400 to $750 \mathrm{~nm}$ after normalizing $\kappa_{\lambda}$ and $\sigma_{s, \lambda}$ by their respective mass concentrations $\left(X_{3, i}\right)_{1 \leq i \leq 3}$ according to Eq. (2). It is evident that the three datasets collapsed on a single line. van de Hulst [44] recommended that a "simple and conclusive test for the absence of multiple scattering" consists of verifying that the scattering intensity is directly

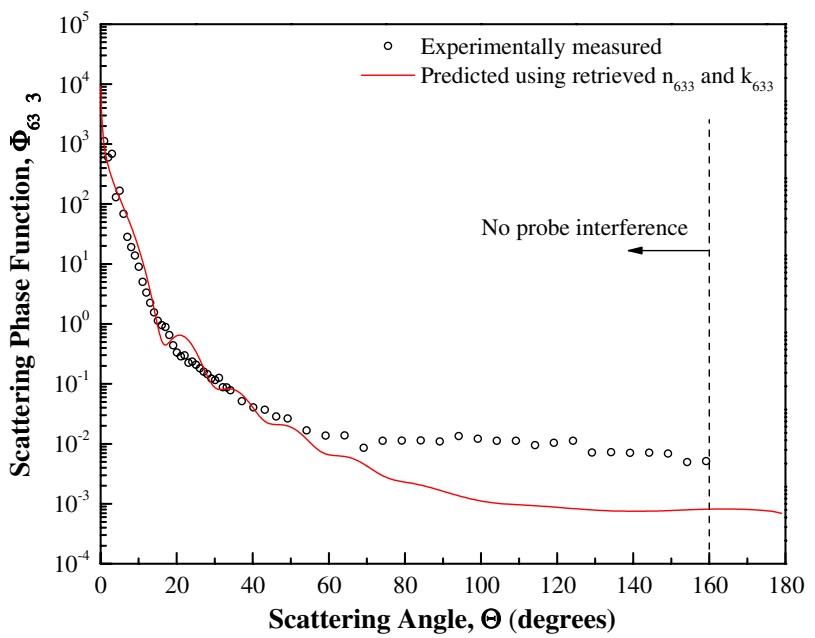

Fig. 4. Scattering phase function of A. cylindrica at $633 \mathrm{~nm}$ measured experimentally using a polar nephelometer and predicted using the retrieved values of $n_{633}$ and $k_{633}$ for Sample 3 with $x_{w}=0.75$. 

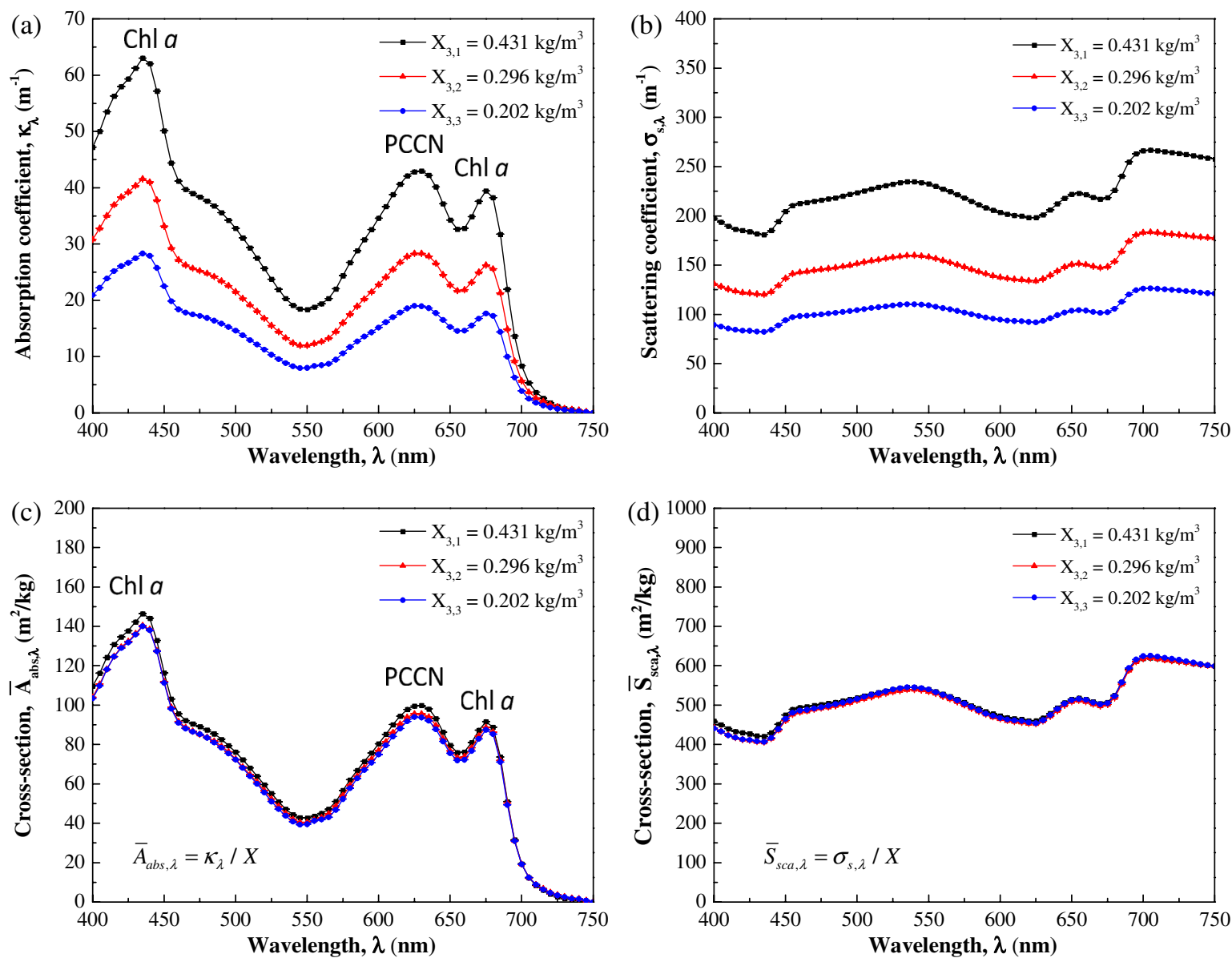

Fig. 5. (a) Absorption coefficient, $\kappa_{\lambda}$, (b) scattering coefficient, $\sigma_{s, \lambda}$, (c) average mass absorption cross section, $\bar{A}_{\text {abs }, \lambda}=\kappa_{\lambda} / X$, and (d) average mass scattering cross section, $\bar{S}_{\mathrm{sca}, \lambda}=\sigma_{s, \lambda} / X$, from 400 to $750 \mathrm{~nm}$ of $A$. cylindrica in Sample 3 for mass concentrations, $X_{3,1}=0.431, X_{3,2}=0.296$, and $X_{3,3}=0.202 \mathrm{~kg} / \mathrm{m}^{3}$.

proportional to particle concentration. Therefore, the experimental measurements confirm that single and independent scattering prevailed (Assumption 2) and that absorption and scattering were linear processes, as suggested by Eq. (2). In addition, scattering dominated over absorption at all wavelengths between 400 and $750 \mathrm{~nm}$. Similar results were obtained for the absorption and scattering coefficients, $\kappa_{\lambda}$ and $\sigma_{s, \lambda}$, and for the average mass absorption and scattering cross sections, $\bar{A}_{\text {abs }, \lambda}$ and $\bar{S}_{\text {sca, } \lambda}$, measured at other times during the growth.

Figure $\underline{6}$ presents the average (a) mass absorption, $\bar{A}_{\text {abs. } \lambda}$, and (b) scattering, $\bar{S}_{\text {sca. } \lambda}$, cross sections over the PAR region for Samples 1 to 4 collected after 6, 9, 12, and 14 days during batch culture. From day 6 (Sample 1) to day 9 (Sample 2), the absorption cross section at $480 \mathrm{~nm}, \bar{A}_{\text {abs.480 }}$, decreased but did not change significantly afterward. Simultaneously, both $\bar{A}_{\text {abs.630 }}$ and $\bar{A}_{\text {abs. } 676}$ increased. This can be attributed to the adaptation of the microorganisms to the decreasing average fluence rate in the culture bottle caused by increasing biomass concentrations. As a response, they decrease their PPC concentration and increase their Chl $a$ and PCCN concentrations [7]. After day 9, the absorption cross section, $\bar{A}_{\text {abs } 630}$, corresponding to PCCN, decreased significantly while $\bar{A}_{\text {abs. } 676}$ decreased slightly. The sharp decrease in $\bar{A}_{\text {abs, } 630}$ can be attributed to the selective degradation of PCCN caused by the nitrogen deficient conditions prevailing after 9 days. In fact, PCCN degradation has been suggested as a mechanism to provide endogenously amino acids for the maintenance and synthesis of nitrogenase $[\underline{45}, \underline{46}]$. This degradation was found to be selective and did not affect significantly the cells' Chl $a$ content in other cyanobacteria including (i) unicellular [46] and (ii) filamentous nonheterocystous [47]. Finally, note that the absorption peak at $436 \mathrm{~nm}$ was affected by both Chl $a$ and carotenoids and decreased continuously from days 6 to 14 . This can be explained by the successive decrease in carotenoid concentration between days 6 and 9 and in Chl $a$ concentration from days 9 to 14 .

Moreover, the average mass scattering cross sections for all samples were identical at $750 \mathrm{~nm}$. This was due to Assumption 5 stating that $\bar{S}_{\text {sca. } 750}$ remained constant during the growth. Hence, the same mass calibration curve [Eq. (5)] was used to determine $X$ for all samples. This method of estimating the mass concentration of photosynthetic microorganisms has been widely used [48]. However, the cells of the cyanobacterium undergo compositional and morphological changes during its growth phase, which may cause $\bar{S}_{\mathrm{sca}, \lambda}$ to vary over time. Overall, the relative difference in $\bar{S}_{\text {sca }, \lambda}$ among the four samples was less than $13 \%$ over the entire PAR region. This relatively small difference suggests that assuming $\bar{S}_{\text {sca.750 }}$ to be constant did not introduce significant errors in the measurements of $X$ with respect to time from OD measurements at $750 \mathrm{~nm}$. In other words, changes in cell composition and 

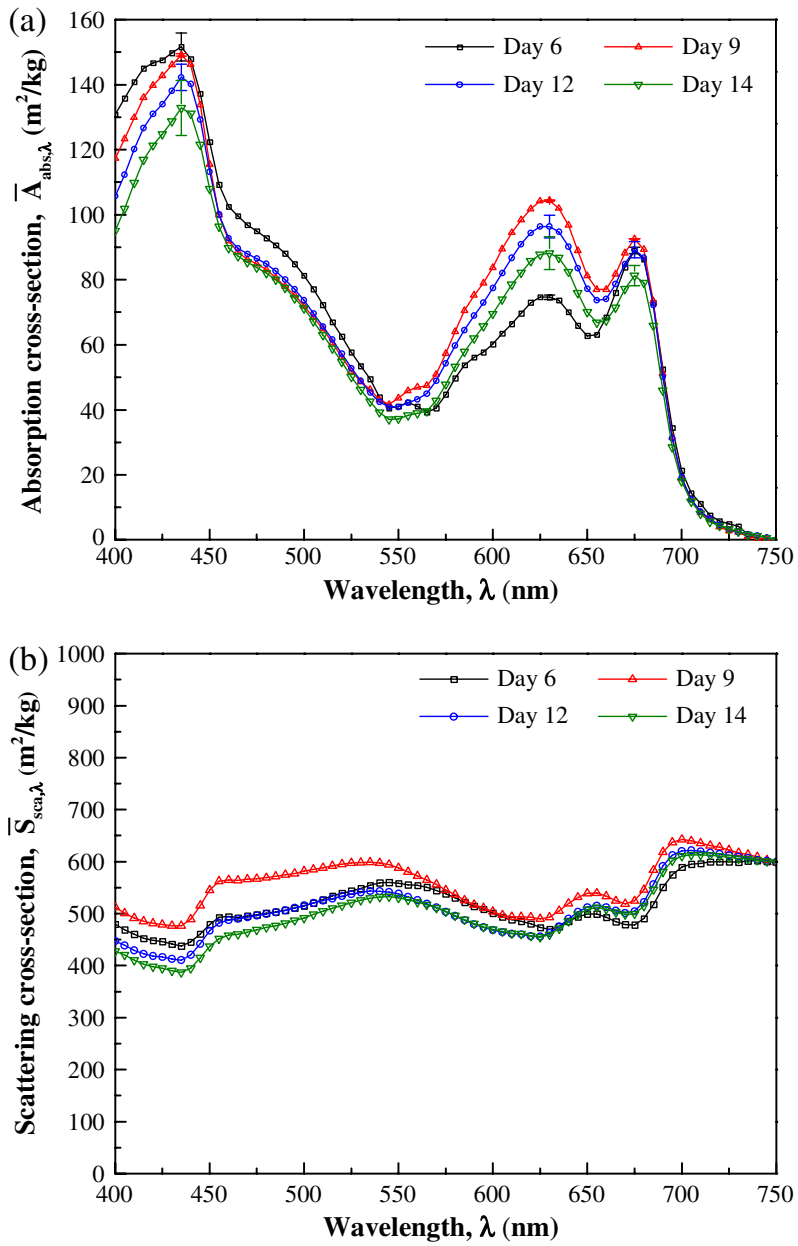

Fig. 6. Average mass (a) absorption, $\bar{A}_{\text {abs }, \lambda}$, and (b) scattering cross sections $\bar{S}_{\mathrm{sca},}$ from 400 to $750 \mathrm{~nm}$ of $A$. cylindrica for Samples 1, 2, 3, and 4 collected after day $6,9,12$, and 14 , respectively.

heterocyst frequency were not sufficient to affect significantly the scattering cross section.

Finally, Berberoğlu and Pilon [12] reported the mass absorption and scattering cross sections of filamentous Anabaena variabilis grown in ATCC medium 616 under 2000-3000 lux of fluorescent white light. Comparatively, $A$. cylindrica featured values of $\bar{A}_{\mathrm{abs}, \lambda}$ and $\bar{S}_{\mathrm{sca}, \lambda}$ that were approximately twice smaller than those of $A$. variabilis for all wavelengths in the PAR region. This is likely due to the fact that $A$. variabilis had a larger cell diameter, around $5 \mu \mathrm{m}$, compared with $2.7 \mu \mathrm{m}$ for A. cylindrica. It could also be due to different pigment concentrations even though both species are filamentous cyanobacteria from the same genus.

\section{Effective Optical Properties}

\section{Validation of the Inverse Method}

The scattering cross sections of $99 \%$ silica glass fibers more than $1 \mathrm{~mm}$ long, supplied by OMEGA Engineering, Inc., USA (Part No: XS-K-24SLE), were measured between 400 and $725 \mathrm{~nm}$ using the same experimental setup and procedure as those used to measure $\bar{C}_{\mathrm{abs}, \lambda}^{\prime}$ and $\bar{C}_{\mathrm{sca}, \lambda}^{\prime}$ for $A$. cylindrica. The fiber size distribution was also measured using 113 fibers and varied between 4 and $8 \mu \mathrm{m}$ in diameter with a mean diameter of $5.88 \mu \mathrm{m}$ and standard deviation of $0.65 \mu \mathrm{m}$. Note that, in the visible part of the spectrum, the absorption index of silica glass is less than $2 \times 10^{-7}$ and was taken as $k_{\lambda}=0$ [ [49]. The real part of the complex index of refraction, $n_{\lambda}$, of the glass fibers was retrieved over the PAR region using the inverse method previously described. The results were compared with predictions from the following three-term Sellmeier equation, valid between 0.21 and $6.7 \mu \mathrm{m}$ [노, $\underline{50}$ ],

$$
\begin{aligned}
n_{\lambda}^{2}= & 1+\frac{0.6961663 \lambda^{2}}{\lambda^{2}-(0.0684043)^{2}}+\frac{0.4079426 \lambda^{2}}{\lambda^{2}-(0.1162414)^{2}} \\
& +\frac{0.8974794 \lambda^{2}}{\lambda^{2}-(9.896161)^{2}},
\end{aligned}
$$

where $\lambda$ is expressed in $\mu$ ms. The maximum and average relative differences over the PAR region were $4 \%$ and $2 \%$, respectively (see Supplementary Materials). These results validate both the experimental and theoretical methods used in this study.

\section{Cylindrica}

Figure 7 shows (a) the refraction index, $n_{\lambda}$, and (b) the absorption index, $k_{\lambda}$, of $A$. cylindrica between 400 and $750 \mathrm{~nm}$ for Samples 1, 2, 3, and 4 retrieved from (i) the measured average mass absorption and scattering cross sections, (ii) the volumeequivalent cylinder diameter distribution, $f\left(d_{c, e q}\right)$, and (iii) for
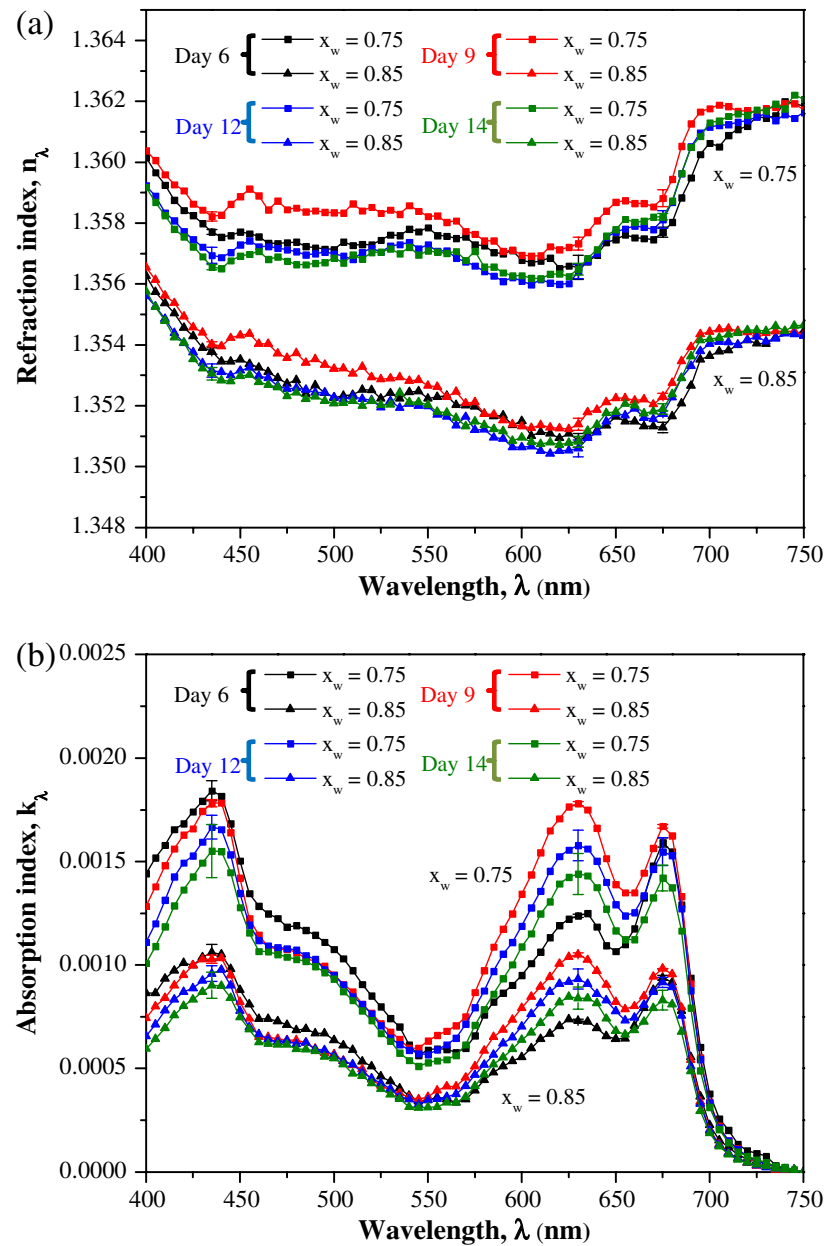

Fig. 7. Retrieved (a) refraction, $n_{\lambda}$, and (b) absorption, $k_{\lambda}$, indices between 400 to $750 \mathrm{~nm}$ for A. cylindrica assuming water mass fraction to be $x_{w}=0.75$ or 0.85 for Samples $1,2,3$, and 4 collected after day $6,9,12$, and 14 , respectively. 
water mass fractions, $x_{w}$, equal to 0.75 or 0.85 . The error bars were estimated from error propagation analysis at wavelengths 436, 630, and $676 \mathrm{~nm}$.

First, it is worth noting that $n_{\lambda}$ and $k_{\lambda}$, retrieved for each of the 71 individual wavelengths considered, were continuous functions of wavelengths. Second, $k_{\lambda}$ featured peaks at 436 , 630, and $676 \mathrm{~nm}$ corresponding to in vivo absorption peaks Chl $a$ and PPCN, the pigments of A. cylindrica [28,29]. Third, the values retrieved for $n_{\lambda}$ and $k_{\lambda}$ were within the ranges of those found for microalgae $[10,20]$ and other cyanobacteria previously reported in the literature [20,22]. These results could not have been guessed a priori as they were not intrinsic consequences of the inverse method, which retrieved $n_{\lambda}$ and $k_{\lambda}$ for each wavelength independently. This further brings confidence in the inverse method.

The refraction index, $n_{\lambda}$, retrieved did not vary significantly with time. In addition, the values obtained with $x_{w}=0.75$ were less than $1 \%$ larger than that obtained with $x_{w}=0.85$ for all samples and across the PAR region. In other words, $n_{\lambda}$ was not sensitive to water mass fraction $x_{w}$. Small dips in $n_{\lambda}$ were observed around wavelengths corresponding to absorption peaks in $k_{\lambda}$. This can be attributed to oscillator resonances predicted from optical constant theory such as the Lorenz model [42] or the Ketteler-Helmholtz theory [22], for example. By contrast, the absorption index, $k_{\lambda}$, displayed large sensitivity to the chosen value of $x_{w}$.

Finally, the scattering phase function, $\Phi_{T, \lambda}\left(\hat{\mathbf{s}}_{i}, \hat{\mathbf{s}}\right)$, in the PAR region was computed by (i) treating $A$. cylindrica as infinitely long and randomly oriented cylinders with volume-equivalent size distribution and (ii) using the values of $n_{\lambda}$ and $k_{\lambda}$ retrieved assuming water mass fraction $x_{w}$ of 0.75 and 0.85 . The associated asymmetry factor at $633 \mathrm{~nm}$ for samples 1-4 was found to range between 0.983 and 0.985 . These results are in good agreement with the value of 0.985 obtained experimentally, as illustrated in Fig. 4. Note that at a scattering angle, $\Theta$, larger than $60^{\circ}$, the experimentally measured phase function showed significant differences with the predicted phase function. This may be attributed to the fact that filamentous $A$. cylindrica were treated as infinitely long cylinders. In fact, similar differences in the phase functions of linear chains of spheres and volume-equivalent infinitely long cylinders with large size parameters can be observed in data reported by Lee and Pilon [32]. Note also that, for the microorganism considered, the size parameter is large, scattering is strongly forward, and only a small amount of energy is contained in the radiation scattered in scattering angles beyond $60^{\circ}$. The asymmetry factor and the scattering phase function varied very slightly from one sample to another and with the choice of water mass fraction, $x_{w}$. In fact, they depended mainly on the size distribution and on the index of refraction, which remained nearly constant during the course of the experiments. Finally, the predicted scattering phase function was practically independent of wavelength over the PAR region. These results confirmed Assumption 4 used to retrieve the absorption and scattering cross sections.

\section{CONCLUSION}

This paper presented the mass absorption and scattering cross sections and the complex index of refraction of $A$. cylindrica from 400 to $750 \mathrm{~nm}$ at different times during batch growth in a nitrate-free medium under 3000 lux of white fluorescent light. An inverse method was developed to retrieve the real and imaginary parts of the complex index of refraction from the measured scattering phase function and average mass scattering and absorbing cross sections. It treated A. cylindrica filaments as homogeneous, randomly oriented, and infinitely long volume-equivalent cylinders. $A$. cylindrica scattered mainly in the forward direction due to its large size parameter. The absorption index and absorption cross section showed distinct absorption peaks at 436 and $676 \mathrm{~nm}$, corresponding to chlorophyll $a$, and at 480 and $630 \mathrm{~nm}$, corresponding to carotenoids and PCCN, respectively. Absorption peaks corresponding to Chl $a$ and PCCN increased and those of PPC decreased from day 6 to day 9 , due to photoacclimation in response to low fluence rate in the PBR caused by increasing biomass concentration. The absorption peaks corresponding to PCCN concentrations decreased sharply after day 9. This was attributed to the degradation of PCCN as an endogenous nitrogen source to maintain and to synthesize nitrogenase enzyme during nitrogen starvation. This interpretation was corroborated by the simultaneous increase in heterocyst frequency. Finally, unlike the refraction index, $n_{\lambda}$, absorption index $k_{\lambda}$ varied during the batch culture and was very sensitive to the water mass fraction, $x_{w}$, used in the inverse method. The results can be used to predict and optimize light distribution in photobioreactors growing A. cylindrica for wastewater treatment, ammonia, or hydrogen production. The methodology can also be applied to a wide range of scattering suspensions containing cylindrical scatterers.

\section{REFERENCES}

1. M. L. Parry, ed., Climate Change 2007: Impacts, Adaptation and Vulnerability. Contribution of Working Group II to the Fourth Assessment Report of the Intergovernmental Panel on Climate Change (IPPC) (Cambridge University, 2007), Vol. 4.

2. Y. Chisti, "Biodiesel from microalgae," Biotechnol. Adv. 25, 294-306 (2007).

3. L. Pilon, H. Berberoğlu, and R. Kandilian, "Radiation transfer in photobiological carbon dioxide fixation and fuel production by microalgae,” J. Quant. Spectrosc. Radiat. Transfer 112, 2639 2660 (2011).

4. D. Das and T. N. Veziroğlu, "Hydrogen production by biological processes: a survey of literature," Int. J. Hydrogen Energy 26, 13-28 (2001).

5. A. Richmond, Handbook of Microalgal Culture (Blackwell Science, 2004).

6. J. R. Benemann, "Production of nitrogen fertilizer with nitrogenfixing blue-green algae," Enzyme Microb. Technol. 1, 83-90 (1979).

7. P. G. Falkowski and J. LaRoche, "Acclimation to spectral irradiance in algae," J. Phycol. 27, 8-14 (1991).

8. B. Ke, Photosynthesis, Photobiochemistry and Photobiophysics (Kluwer Academic, 2001).

9. J.-F. Cornet, C. G. Dussap, and G. Dubertret, "A structured model for simulation of cultures of the cyanobacterium Spirulina platensis in photobioreactors: I. Coupling between light transfer and growth kinetics," Biotechnol. Bioeng. 40, 817-825 (1992).

10. E. Lee, R.-L. Heng, and L. Pilon, "Spectral optical properties of selected photosynthetic microalgae producing biofuels," J. Quant. Spectrosc. Radiat. Transfer 114, 112-135 (2013).

11. M. F. Modest, Radiative Heat Transfer, 2nd edition (Academic, 2003).

12. H. Berberoğlu and L. Pilon, "Experimental measurement of the radiation characteristics of Anabaena variabilis ATCC 29413-U and Rhodobacter sphaeroides ATCC 49419," Int. J. Hydrogen Energy 32, 4772-4785 (2007). 
13. H. Berberoğlu, L. Pilon, and A. Melis, "Radiation characteristics of Chlamydomonas reinhardtii CC125 and its truncated chlorophyll antenna transformants tla1, tlaX, and tla1-CW', Int. J. Hydrogen Energy 33, 6467-6483 (2008).

14. H. Berberoğlu, P. S. Gomez, and L. Pilon, "Radiation characteristics of Botryococcus braunii, Chlorococcum littorale, and Chlorella $s p$. used for $\mathrm{CO}_{2}$ fixation and biofuel production," J. Quant. Spectrosc. Radiat. Transfer 110, 1879-1893 (2009).

15. D. Stramski and C. D. Mobley, "Effect of microbial particles on oceanic optics: a database of single-particle optical properties," Limnol. Oceanogr. 42, 538-549 (1997).

16. A. Quirantes and S. Bernard, "Light scattering by marine algae: two-layer spherical and nonspherical models," J. Quant. Spectrosc. Radiat. Transfer 89, 311-321 (2004).

17. L. Pottier, J. Pruvost, J. Deremetz, J. F. Cornet, J. Legrand, and C. G. Dussap, "A fully predictive model for one-dimensional light attenuation by Chlamydomonas reinhardtii in a torous photobioreactor," Biotechnol. Bioeng. 91, 569-582 (2005).

18. R. Kandilian, E. Lee, and L. Pilon, "Radiation and optical properties of Nannochloropsis oculata grown under different irradiances and spectra," Bioresour. Technol. 137, 63-73 (2013).

19. P. M. Slegers, R. H. Wijffels, G. van Straten, and A. J. B. van Boxtel, "Design scenarios for flat panel photobioreactors," Appl. Energy 88, 3342-3353 (2011).

20. A. Morel and A. Bricaud, "Inherent optical properties of algal cells including picoplankton: theoretical and experimental results," Can. Bull. Fish. Aquat. Sci. 214, 521-560 (1986).

21. Z. Dubinsky and N. Stambler, "Photoacclimation processes in phytoplankton: mechanisms, consequences, and applications," Aquat. Microb. Ecol. 56, 163-176 (2009).

22. M. Jonasz and G. R. Fournier, Light Scattering by Particles in Water: Theoretical and Experimental Foundations (Academic, 2007).

23. P. Fay, "Photostimulation of nitrogen fixation in Anabaena cylindrica," Biochim. Biophys. Acta. Bioenerg. 216, 353-356 (1970).

24. N. M. Weare and J. R. Benemann, "Nitrogen fixation by Anabaena cylindrica," Arch. Microbiol. 90, 323-332 (1973).

25. W. D. P. Stewart and P. Rowell, "Effects of l-methionine-dlsulphoximine on the assimilation of newly fixed $\mathrm{NH}_{3}$, acetylene reduction and heterocyst production in Anabaena cylindrica," Biochem. Biophys. Res. Commun. 65, 846-856 (1975).

26. M. J. Griffiths and S. Harrison, "Lipid productivity as a key characteristic for choosing algal species for biodiesel production," Appl. Environ. Microbiol. 33, 123-131 (1977).

27. L. Vasconcelos and P. Fay, "Nitrogen metabolism and ultrastructure in Anabaena cylindrica," Arch. Microbiol. 96, 271-279 (1974).

28. P. Fay, "Cell differentiation and pigment composition in Anabaena cylindrica," Arch. Microbiol. 67, 62-70 (1969).

29. R. Bidigare, M. Ondrusek, J. Morrow, and D. Kiefer, "In vivo absorption properties of algal pigments," Proc. SPIE 1302 290-302 (1990).

30. C. P. Wolk and R. D. Simon, "Pigments and lipids of heterocysts," Planta 86, 92-97 (1969).

31. P. Charbonneau and B. Knapp, "A user's guide to PIKAIA 1.0," Tech. Rep. NCAR Technical Note 418+IA (National Center for Atmospheric Research, 1995).
32. E. Lee and L. Pilon, "Light scattering by long and randomly oriented linear chains of spheres," J. Opt. Soc. Am. A 30, 1892-1900 (2013).

33. T. R. Parsons, K. Stephens, and J. D. H. Strickland, "On the chemical composition of eleven species of marine phytoplanktons,” J. Fish. Res. Board Can. 18, 1001-1016 (1961).

34. P. J. l. B. Williams and L. M. L. Laurens, "Microalgae as biodiesel and biomass feedstocks: review and analysis of the biochemistry, energetics and economics," Energ. Environ. Sci. 3, 554-590 (2010).

35. P. A. Roger, A. Tirol, S. Ardales, and I. Watanabe, "Chemical composition of cultures and natural samples of $\mathrm{N}_{2}$-fixing blue-green algae from rice fields," Biol. Fertil. Soils 2, 131-146 (1986).

36. D. C. Sigee, J. Teper, and E. Levado, "Elemental composition of the cyanobacterium Anabaena flos-aquae collected from different depths within a stratified lake," Eur. J. Phycol. 34, 477-485 (1999).

37. K. G. Privoznik, K. J. Daniel, and F. P. Incropera, "Absorption, extinction and phase function measurements for algal suspensions of Chlorella pyrenoidosa," J. Quant. Spectrosc. Radiat. Transfer 20, 345-352 (1978).

38. R. J. Davies-Colley, R. D. Pridmore, and J. E. Hewitt, "Optical properties and reflectance spectra of 3 shallow lakes obtained from a spectrophotometric study," New Zeal. J. Mar. Freshwater Res. 17, 445-459 (1983).

39. J. Dauchet, "Analyse Radiative des Photobioréacteurs," Ph.D. dissertation, (Université Blaise Pascal, Clermont Ferrand II, France, 2012).

40. O. Zhernovaya, O. Sydoruk, V. Tuchin, and A. Douplik, "The refractive index of human hemoglobin in the visible range," Phys. Med. Biol. 56, 4013-4021 (2011).

41. M. Kerker, The Scattering of Light, and Other Electromagnetic Radiation (Academic, 1969).

42. C. F. Bohren and D. R. Huffman, Absorption and Scattering of Light by Small Particles (Wiley, 1998).

43. P. F. Brownell and D. J. D. Nicholas, "Some effects of sodium on nitrate assimilation and $\mathrm{N}_{2}$ fixation in Anabaena cylindrica," Plant Physiol. 42, 915-921 (1967).

44. H. C. van de Hulst, Light Scattering by Small Particles (Courier Dover, 2012)

45. M. M. Allen and A. J. Smith, "Nitrogen chlorosis in blue-green algae," Arch. Microbiol. 69, 114-120 (1969).

46. M. M. Allen and F. Hutchison, "Nitrogen limitation and recovery in the cyanobacterium Aphanocapsa 6308," Arch. Microbiol. 128, 1-7 (1980).

47. I. Canto de Loura, J. P. Dubacq, and J. C. Thomas, "The effects of nitrogen deficiency on pigments and lipids of cyanobacteria," Plant Physiol. 83, 838-843 (1987).

48. M. J. Griffiths, C. Garcin, R. P. van Hille, and S. T. Harrison, "Interference by pigment in the estimation of microalgal biomass concentration by optical density," J. Microbiol. Methods 85, 119-123 (2011).

49. R. Kitamura, L. Pilon, and M. Jonasz, "Optical constants of silica glass from extreme ultraviolet to far infrared at near room temperature," Appl. Opt. 46, 8118-8133 (2007).

50. I. H. Malitson, "Interspecimen comparison of the refractive index of fused silica," J. Opt. Soc. Am. 55, 1205-1209 (1965). 